\title{
MONOFUNCTORS AS REFLECTORS
}

\author{
BY \\ CLAUS MICHAEL RINGEL $\left({ }^{1}\right)$
}

\begin{abstract}
In a well-powered and co-well-powered complete category $\mathscr{X}$ with weak amalgamations, the class $M$ of all reflective subcategories with a monofunctor as refiector forms a complete lattice; the limit-closure of the union of any class of elements of $\boldsymbol{M}$ belongs to $\boldsymbol{M}$. If $\mathscr{X}$ has injective envelopes, then the set-theoretical intersection of any class of elements of $\boldsymbol{M}$ belongs to $\boldsymbol{M}$.
\end{abstract}

1. Introduction. Let $\mathscr{K}$ be a well-powered and co-well-powered complete category. It is well known that for all limit-closed subcategories $\left({ }^{2}\right) \mathscr{X}$ of $\mathscr{K}$ the full subcategory $s \mathscr{X}$ of all subobjects of objects of $\mathscr{X}$ is a reflective subcategory of $\mathscr{K}$. We say that $\mathscr{K}$ has amalgamations $\left({ }^{3}\right)$ iff for any two monomorphisms $m_{i}: A \rightarrow B_{i}(i=1,2)$ there is a commutative square of the form

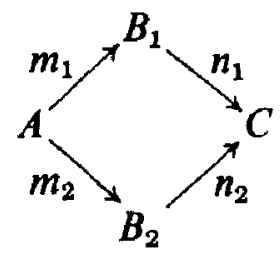

where $n_{1}$ and $n_{2}$ are monomorphisms. If we assume that $\mathscr{K}$ has amalgamations, then any limit-closed subcategory $\mathscr{X}$ with s $\mathscr{X}=\mathscr{K}$ is reflective; more generally, a limit-closed subcategory $\mathscr{X}$ for which the reflector of $\mathscr{K}$ onto $\mathbf{S} \mathscr{X}$ is a monofunctor is reflective and has itself a monofunctor as reflector (Theorem 1). We denote by $\boldsymbol{M}$ the class of all reflective subcategories with a monofunctor as reflector. Thus a limit-closed subcategory $\mathscr{X}$ belongs to $M$ if and only if $s \mathscr{X}$ belongs to $M . M$ is partially ordered by inclusion and has a smallest element. We show that the limitclosure of the union of any class of elements of $M$ belongs to $M$ (Theorem 2); therefore $\boldsymbol{M}$ is a complete lattice. A category with injective envelopes always has amalgamations. In these categories the set-theoretical intersection of any class of members of $\boldsymbol{M}$ also belongs to $\boldsymbol{M}$ (Theorem 4).

Received by the editors November 18, 1970.

AMS 1970 subject classifications. Primary 18A40; Secondary 18A20, 18E40, 18F10.

Key words and phrases. Category with amalgamations, lattice of reflective subcategories, limit-closure of the injectives, injective envelope, locally minimal subcategories, locally maximal subcategories, torsion theories in a module category, Grothendieck topologies.

(1) This work was partially supported by a grant of the NRC of Canada.

${ }^{(2)}$ In this paper "subcategory" always means full and replete subcategory.

( ${ }^{3}$ In the terminology of [3], $\mathscr{X}$ has weak amalgamations. 
In the category of all modules over a ring $R$ a limit-closed subcategory $X$ belongs to $M$ iff there is a torsion-theory $(\mathscr{P}, \mathscr{2})$ with $s \mathscr{X}=\mathscr{2}$. If $\mathscr{X}$ is the functorcategory $[\mathscr{C}, \subseteq]$, where $\mathscr{C}$ is small and $\Xi$ is the category of sets, then $\mathscr{X}$ belongs to $M$ iff $\mathrm{s} X$ is the category of all separated presheaves with respect to some Grothendiecktopology.

2. The reflectivity of limit-closed subcategories. The following remark will be useful in the sequel. In a category with amalgamations every bimorphism is an essential monomorphism. For given a bimorphism $b$ and a morphism $x$ such that $x b$ is a monomorphism, we find a commuting square

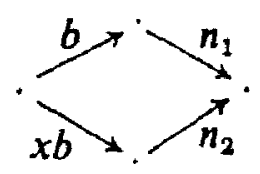

where $n_{1}$ and $n_{2}$ are monomorphisms. But $b$ is an epimorphism, therefore the equality $n_{1} b=n_{2} x b$ implies $n_{1}=n_{2} x$. So $x$ is a monomorphism.

LEMMA 1. Let $\mathscr{K}$ be a well-powered and complete category with amalgamations. If $\mathscr{X}$ is a limit-closed subcategory and $\mathrm{s} \mathscr{X}=\mathscr{K}$, then $\mathscr{X}$ is reflective, and has a monofunctor as reflector.

Proof. For any object $K$ of $\mathscr{K}$ there is a monomorphism $m: K \rightarrow X$ with $X$ in $\mathscr{X}$. Let $m$ factorize as

$$
K \stackrel{m^{\prime}}{\rightarrow} X^{\prime} \stackrel{m^{\prime \prime}}{\longrightarrow} X
$$

where $m^{n}$ is the smallest subobject of $X$ which lies in $X$ and through which $m$ factorizes.

(a) $m^{\prime}$ is a bimorphism. We only have to show that $m^{\prime}$ is an epimorphism. Given $x_{i}: X^{\prime} \rightarrow K^{\prime}$ with $x_{1} m^{\prime}=x_{2} m^{\prime}$ we find a monomorphism $u: K^{\prime} \rightarrow X^{\prime \prime}$ with $X^{n}$ in $\mathscr{X}$. The equalizer $e$ of $u x_{1}$ and $u x_{2}$ belongs to $\mathscr{X}$, and $m^{\prime}$ can be factorized through $e$, therefore $e$ must be an isomorphism. But $u x_{1}=u x_{2}$ implies $x_{1}=x_{2}$, because $u$ is a monomorphism.

(b) Every morphism $a: K \rightarrow Y$ with $Y$ in $\mathscr{X}$ may be factorized through $\boldsymbol{m}^{\prime}$. Let $P$ be the product of $X^{\prime}$ and $Y$ with the projections $p_{1}: P \rightarrow X^{\prime}$ and $p_{2}: P \rightarrow Y$ and define $z: K \rightarrow P$ by $p_{1} z=m^{\prime}$ and $p_{2} z=a$. The last equality implies that $z$ is a monomorphism. $P$ is an object of $\mathscr{X}$, therefore $z$ factorizes as $K \stackrel{z^{\prime}}{\longrightarrow} P^{\prime} \stackrel{z^{\prime \prime}}{\longrightarrow} P$ where $z^{\prime \prime}$ is the smallest subobject of $P$ which belongs to $\mathscr{X}$ and through which $z$ can be factorized. In (a) we have seen that $z^{\prime}$ is a bimorphism and therefore an essential monomorphism. But

$$
\left(p_{1} z^{\prime \prime}\right) z^{\prime}=m^{\prime}
$$

is a monomorphism, therefore $p_{1} z^{\prime \prime}$ is a monomorphism. The equality $\left(^{*}\right)$ also shows that $p_{1} z^{\prime \prime}$ is a subobject through which $m^{\prime}$ factors. Because $p_{1} z^{\prime \prime}$ belongs to $\mathscr{X}$, 
$p_{1} z^{\prime \prime}$ must be an isomorphism. If we define $a^{\prime}=p_{2} z^{\prime \prime}\left(p_{1} z^{\prime \prime}\right)^{-1}$ we get the equality

$$
a^{\prime} m^{\prime}=p_{2} z^{\prime \prime}\left(p_{1} z^{\prime \prime}\right)^{-1}\left(p_{1} z^{\prime \prime} z^{\prime}\right)=p_{2} z^{\prime \prime}\left(p_{1} z^{\prime \prime}\right)^{-1}\left(p_{1} z^{\prime \prime}\right) z^{\prime}=p_{2} z^{\prime \prime} z^{\prime}=a \text {. }
$$

(c) $m^{\prime}$ is a reflection of $K$ into $\mathscr{X}$. In (b) we have seen that every morphism $a: K \rightarrow Y$ with $Y$ in $\mathscr{X}$ may be factorized through $m^{\prime}$ and according to (a) this factorization is unique, because $m^{\prime}$ is an epimorphism.

(d) The reflector of $\mathscr{X}$ onto $\mathscr{X}$ is a monofunctor. Let $k: A \rightarrow B$ be a monomorphism in $\mathscr{X}$. The reflections $a$ of $A$ and $b$ of $B$ define a commutative square

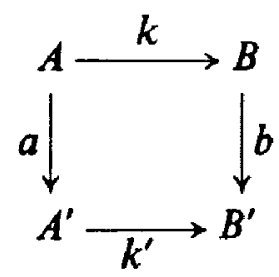

and $k^{\prime}$ is the image of $k$ under the reflector $\mathscr{K} \rightarrow \mathscr{X}$. But $k$ and $b$ are monomorphisms and $a$ is an essential monomorphism, so $k^{\prime}$ is a monomorphism.

REMARK 1. The proof discloses a special feature of categories with amalgamations. We have constructed the reflection by taking any monomorphism $m: K \rightarrow X$ with $X$ in $\mathscr{X}$ and factorizing through a subobject of $X$. The subcategory $\bar{\Re}$ of the compact spaces of the category $\mathfrak{B}$ of all completely regular Hausdorff spaces gives an example where the reflections are bimorphisms but not essential monomorphisms.

REMARK 2. In the proof of Lemma 1 we did not need the existence of infinite products. Also the existence of finite products may be avoided if $\mathscr{K}$ has pushouts: the assertion of Lemma 1 remains true if $\mathscr{H}$ is a well-powered category with arbitrary intersections, pullbacks, pushouts and amalgamations. We have only to change the proof of (b). Instead of forming the product of $X^{\prime}$ and $Y$ we take a pushout $\bar{a}: X^{\prime} \rightarrow Q$ and $\bar{m}: Y \rightarrow Q$ of $a$ and $m$ and a monomorphism $u: Q \rightarrow X^{\prime \prime}$ with $X^{n}$ in $\mathscr{X}$. If in the following diagram $p_{1}$ and $p_{2}$ are pullback-morphisms with respect to $u \bar{a}$ and $u \bar{m}$, we get a morphism $z$ with $p_{1} z=m^{\prime}$ and $p_{2} z=a$.

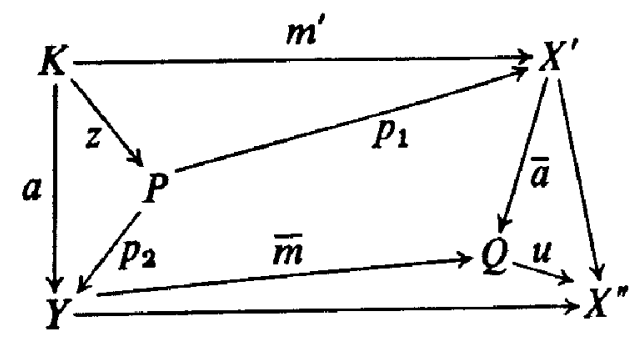

The object $P$ belongs to $\mathscr{X}$ (because $X^{\prime}, Y$ and $X^{\prime \prime}$ are in $\mathscr{X}$ ), therefore $P, p_{1}, p_{2}, z$ have all properties we need for continuing the proof of (b).

In a well-powered and co-well-powered complete category $\mathscr{K}$ every subcategory which is limit-closed and subobject-closed is reflective [1], [5], [8]. In particular, 
if $\mathscr{X}$ is any limit-closed subcategory, s $\mathscr{X}$ is a reflective subcategory of $\mathscr{K}$, and $\mathscr{X}$ is reflective in $\mathscr{K}$ if and only if $\mathscr{X}$ is reflective in s $\mathscr{X}$. With $\mathscr{K}$ also s $\mathscr{X}$ is wellpowered and complete. In order to apply Lemma 1 we have to impose a condition onto $\mathrm{s} \mathscr{X}$ which implies that $\mathrm{s} \mathscr{X}$ has amalgamations. If $\mathscr{X}$ has amalgamations and the reflector onto $\mathrm{s} \mathscr{X}$ is a monofunctor, then $\mathrm{s} \mathscr{X}$ has amalgamations: given two monomorphisms $m_{i}: A \rightarrow B_{i}(i=1,2)$ in $s X$, these morphisms are also monomorphisms in $\mathscr{K}$, therefore we find in $\mathscr{K}$ monomorphisms $n_{i}: B_{i} \rightarrow C$ with $n_{1} m_{1}$ $=n_{2} m_{2}$. If $c: C \rightarrow C^{\prime}$ is the reflection of $C$ into $s, c n_{i}$ is the image of $n_{i}$ under the reflector $\mathscr{K} \rightarrow \mathrm{s} \mathscr{X}$. We have assumed that this functor is a monofunctor, so $c n_{1}$ and $c n_{2}$ define an amalgamation of $m_{1}$ and $m_{2}$ in $s X$. This proves the first part of the following theorem:

THEOREM 1. Let $\mathscr{K}$ be a well-powered and co-well-powered complete category with amalgamations. Let $\mathscr{X}$ be a limit-closed subcategory of $\mathscr{X}$. If $\mathrm{s} \mathscr{X}$ has a monofunctor as reflector, then $\mathscr{X}$ is reflective and has a monofunctor as reflector. Conversely, if $\mathscr{X}$ is reflective and has a monofunctor as reflector, $\mathrm{s} \mathscr{X}$ has a monofunctor as reflector.

It remains to show that a reflective subcategory $\mathscr{X}$ has a monofunctor as reflector if and only if $\mathrm{s} \mathscr{X}$ has a monofunctor as reflector. Let $U_{2}: \mathscr{X} \rightarrow \mathrm{s} \mathscr{X}$ be the inclusionfunctor and $S_{1}: \mathscr{H} \rightarrow \mathrm{s} \mathscr{X}, S_{2}: \mathrm{s} \mathscr{X} \rightarrow \mathscr{X}$ be the reflectors. $\mathscr{X}$ is monoreflective in $\mathrm{s} \mathscr{X}\left({ }^{4}\right)$, therefore $S_{1}$ is a subfunctor of $U_{2} S_{2} S_{1}$. As a right adjoint functor $U_{2}$ is a monofunctor, so if $S_{2} S_{1}$ is a monofunctor, also $U_{2} S_{2} S_{1}$ is a monofunctor-but every subfunctor of a monofunctor is a monofunctor, so $S_{1}$ is a monofunctor, s $x$ satisfies the conditions of Lemma 1 (s $\mathscr{X}$ replacing $\mathscr{K}$ ) and therefore $S_{2}$ is also a monofunctor, so $S_{2} S_{1}$ is a monofunctor.

REMARK 3 . It should be noted that there are limit-closed subcategories $\mathscr{X}$ where the reflector $\mathscr{K} \rightarrow \mathrm{s} \mathscr{X}$ is not a monofunctor, but nevertheless $\mathrm{s} \mathscr{X}$ has weak amalgamations (and therefore Lemma 1 can be applied): the subcategory $\mathscr{X}=\mathrm{s} \mathscr{X}=\mathfrak{F}$ of the torsionfree groups in the category (H) of all groups has amalgamations (the free product of two torsionfree groups with amalgamations of a common subgroup is a torsionfree group), but the reflector $\mathscr{H} \rightarrow \mathfrak{F}$ is not a monofunctor (there are groups generated by torsion-elements which contain torsionfree subgroups). We have restricted our attention to subcategories with a monofunctor as reflector (and not to the wider class of reflective subcategories having amalgamations) because of Theorem 2 and Theorem 4. We do not know if the reflective subcategories with amalgamations form a lattice.

(4) $\mathscr{X}$ is a monoreflective (epireflective, bireflective) subcategory of $\mathscr{L}$ means that $\mathscr{X}$ is a reflective subcategory and moreover for every object $L$ in $\mathscr{L}$ the reflection morphism is a monomorphism (epimorphism, bimorphism). The notion of a monoreflective subcategory should not be confused with the concept of a reflective subcategory with a monofunctor as reflector. In fact, in a category with amalgamations every monoreflective subcategory has a monofunctor as reflector, but the converse need not be true. 
3. The lattice $M$. We assume throughout this section that $\mathscr{K}$ is a well-powered and co-well-powered complete category with amalgamations.

Let $\boldsymbol{M}$ denote the class of all reflective subcategories with a monofunctor as reflector. Theorem 1 shows that a limit-closed subcategory $\mathscr{X}$ belongs to $\boldsymbol{M}$ if and only if s $X$ belongs to $\boldsymbol{M}$. $\boldsymbol{M}$ is partially ordered by inclusion and has a unique minimal element: the subcategory of $\mathscr{K}$ which contains just the terminal objects of $\mathscr{K}$.

If $\mathscr{Y}$ is any subcategory of $\mathscr{K}$, the limit-closure $\mathrm{L} \mathscr{Y}$ is defined as the smallest limit-closed subcategory of $\mathscr{X}$ which contains $\mathscr{Y}$.

THEOREM 2. If $\left\{\mathscr{X}_{i} ; i \in I\right\}$ is any class of elements of $M$, and $\mathscr{X}$ is the limit-closure of the union of the $\mathscr{X}_{i}$, then $\mathscr{X}$ is an element of $\boldsymbol{M}$.

REmARK. In general, the limit-closure of a class of reflective subcategories need not be reflective. In a well-powered complete category every limit-closed subcategory $X$ is the limit-closure of the reflective subcategories contained in $\mathscr{X}$ : for every object $X$ in $X$ the limit-closure $\mathrm{L}(X)$ (the smallest limit-closed subcategory which contains $X$ ) is a reflective subcategory--this is a consequence of the special adjoint functor theorem of Freyd [10]. But in [6] Herrlich has constructed a limitclosed subcategory of a well-powered and co-well-powered complete category which is not reflective.

Proof of Theorem 2. We want to show that $\mathrm{s} X$ has a monofunctor as reflector; then the assertion is a consequence of Theorem 1 .

Let $K$ be an object of $\mathscr{K}$, and let $\mathscr{E}=\left\{e_{i}: K \rightarrow E_{i} ; i \in I\right\}$ be the class of reflections of $K$ into the subcategories $\mathrm{s} \mathscr{X}_{i}$.

(a) If $J$ is a subset of the index-class $I$ such that every quotient $e_{i}$ in $\mathscr{E}$ is equivalent to a quotient $e_{j} \mathscr{E}$ with $j$ in $J$, then the reflection of $\mathscr{K}$ into $s \mathscr{X}$ may be constructed in the following way: we form the product $P=\prod_{j \in J} E_{j}$ with the projections $p_{j}: P \rightarrow E_{j}$ and define $f$ by $p_{j} f=e_{j}$. If we factorize $f$ as

$$
K \stackrel{e}{\longrightarrow} E \stackrel{m}{\longrightarrow} P
$$

where $m$ is the smallest subobject of $P$ through which $f$ can be factorized, $e$ is a reflection of $\mathscr{K}$ into $s \mathscr{X}$.

(b) If there is given a monomorphism $k: K \rightarrow K^{\prime}$, then the reflection classes $\mathscr{E}=\left\{e_{i}: K \rightarrow E_{i} ; i \in I\right\}$ and $\mathscr{E}^{\prime}=\left\{e_{i}^{\prime}: K^{\prime} \rightarrow E_{i}^{\prime} ; i \in I\right\}$ both contain only a set of nonequivalent quotients, because $\mathscr{K}$ is co-well-powered. Therefore we find a subset $J$ of $I$ such that every quotient $e_{i}$ in $\mathscr{E}$ is equivalent to a quotient $e_{j}$ with $j \in J$ and every quotient $e_{i}^{\prime}$ in $\mathscr{E}^{\prime}$ is equivalent to a quotient $e_{j}^{\prime}$ with $j \in J$. For every $j \in J$ we define $k_{j}$ by $e_{j}^{\prime} k=k_{j} e_{j} . k_{j}$ is the image of $k$ under the reflector $\mathscr{K} \rightarrow \mathrm{s} \mathscr{T}_{i}$. This is a monofunctor (Theorem 1), therefore the morphisms $k_{f}$ are monomorphisms. The product $\prod_{j \in J} k_{j}: P \rightarrow P^{\prime}$, where $P=\prod_{j \in J} E_{j}$ and $P^{\prime}=\prod_{j \in J} E_{j}^{\prime}$ is a morphism which satisfies the equality $\left(\Pi k_{j}\right) f=f^{\prime} k$ where $f$ and $f^{\prime}$ are defined as in (a). 
Moreover $\prod k_{j}$ is a monomorphism. Factorizing $f=m e$ and $f^{\prime}=m^{\prime} e^{\prime}$ as in (a) gives us the following commutative diagram:

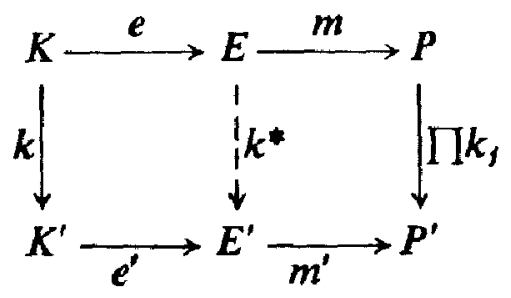

$e$ is an extreme epimorphism, $m^{\prime}$ is a monomorphism, therefore we find $k^{*}$ with $k^{*} e=e^{\prime} k$ and $m^{\prime} k^{*}=\left(\prod k_{j}\right) m$. The first equality shows that $k^{*}$ is the image of $k$ under the reflector $\mathscr{K} \rightarrow \mathrm{s} \mathscr{X}$, the second equality implies that $k^{*}$ is a monomorphism (because $\Pi k_{j}$ and $m$ are monomorphisms).

As a consequence we have the following:

Corollary. $M$ is a complete latice.

Proof. $M$ is partially ordered and has a unique smallest element. In this situation the existence of arbitrary unions in $\boldsymbol{M}$ implies the existence of arbitrary intersections in $\boldsymbol{M}$.

In order to show that the intersections in $M$ are just the set-theoretical intersections of the elements of $\boldsymbol{M}$, we need a new assumption: the existence of injective envelopes. This will be done in the next section.

4. Categories with injective envelopes. Let us remind ourselves of some definitions. An object $I$ is called injective, iff for every monomorphism $m: A \rightarrow B$ and every morphism $a: A \rightarrow I$ there is a morphism $b: B \rightarrow I$ with $b m=a$. A category $\mathscr{K}$ has (enough) injectives, iff for every object $K$ in $\mathscr{K}$ there is a monomorphism $m: K \rightarrow I$ with $I$ injective; $\mathscr{K}$ has injective envelopes iff always such a morphism exists which is an essential monomorphism. It is well known (see for example [3]), that a category with finite products and injectives has amalgamations, so in the sequel we need not mention this assumption.

LEMMA 2. Let $\mathscr{K}$ be a well-powered and co-well-powered complete category. If $\mathcal{I}$ is any class of injective objects of $\mathscr{K}$, then the limit-closure $L$ of $\mathscr{I}$ is reflective subcategory with a monofunctor as reflector.

Proof. We set $\mathscr{X}=\mathrm{L} \mathscr{F}$.

(a) For every object $Y$ in $s \mathscr{X}$ there is a monomorphism $u: Y \rightarrow I$ where $I$ belongs to $\mathscr{X}$ and is injective in $\mathscr{X}$. $Y$ is an object of sL $\mathscr{F}$, therefore a subobject of a product of elements of $\mathscr{I}$. Let $u: Y \rightarrow I$ be a monomorphism where $I$ is a product of elements of $\mathscr{I} . \mathscr{X}$ is the limit-closure of $\mathscr{I}$, so $I$ belongs to $\mathscr{X}$; the elements of $\mathscr{I}$ are injective in $\mathscr{K}$, a product of injective objects is injective, so $I$ is injective in $\mathscr{K}$. 
(b) $\mathrm{s} \mathscr{X}$ has a monofunctor as reflector. Given a monomorphism $m: A \rightarrow B$ in $\mathscr{X}$, we look at the reflections $a$ of $A$ and $b$ of $B$ into $s \mathscr{X}$. There is a morphism $m^{\prime}$ such that the following diagram is commutative

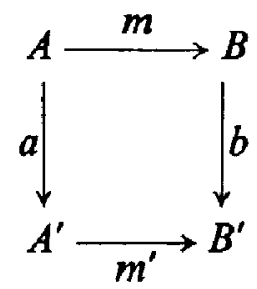

and $m^{\prime}$ is the image under the reflector $\mathscr{X} \rightarrow \mathrm{s} \mathscr{X} . A^{\prime}$ is an object of $\mathrm{s} \mathscr{X}$; according to (a) we find a monomorphism $u: A^{\prime} \rightarrow I$ where $I$ belongs to $\mathscr{X}$ and is injective in $\mathscr{X} . m$ is a monomorphism, $I$ is injective, so there exists a morphism $x$ with $x m=u a . x: B \rightarrow I$ can be factorized through $b$, because $I$ belongs to $s \mathscr{X}: x=x^{\prime} b$. But the equality $u a=x m=x^{\prime} b m=x^{\prime} m^{\prime} a$ implies $u=x^{\prime} m^{\prime}$ (because $a$ is a reflection morphism). $u$ is a monomorphism, so $m^{\prime}$ is a monomorphism.

(c) We want to apply Lemma 1 with $s \mathscr{X}$ instead of $\mathscr{K}$. $\mathrm{s} \mathscr{X}$ is well-powered and complete, (a) implies that $\mathrm{s} \mathscr{X}$ has enough injectives, so $\mathrm{s} \mathscr{X}$ has amalgamations. Therefore $\mathscr{X}$ is a reflective subcategory of $\mathrm{s} \mathscr{X}$ and the reflector of $\mathrm{s} \mathscr{X}$ onto $\mathscr{X}$ is a monofunctor. The reflector of $\mathscr{K}$ onto $\mathscr{X}$ is the composition of the reflector $\mathscr{X} \rightarrow \mathrm{s} \mathscr{X}$ and $\mathrm{s} \mathscr{X} \rightarrow \mathscr{X}$. Both functors are monofunctors, so $\mathscr{X}$ has a monofunctor as reflector.

In the presence of injective envelopes we can characterize the elements of $\boldsymbol{M}$ in the following way:

THEOREM 3. Let $\mathscr{K}$ be a well-powered and co-well-powered complete category with injective envelopes. Then the following assertions are equivalent for a limitclosed subcategory $\mathscr{X}$ :

(i) $\mathscr{X}$ is an element of $\boldsymbol{M}$,

(ii) $\mathscr{X}$ is closed under injective envelopes,

(iii) for every object $X$ in $\mathscr{X}$ there is a monomorphism $X \rightarrow I$ in $\mathscr{X}$, where $I$ is injective in $\mathscr{K}$.

Proof. (i) $\rightarrow$ (ii). Let $m: X \rightarrow I$ be an injective envelope with $X$ in $\mathscr{X}$. If $c: I \rightarrow I^{\prime}$ is the reflection of $I$ in $\mathscr{X}, \mathrm{cm}$ is the image of $m$ under the reflector $S: \mathscr{X} \rightarrow \mathscr{X}$. But $m$ is a monomorphism and $S$ is a monofunctor, so $\mathrm{cm}$ is a monomorphism. $m$ is an essential monomorphism implies that $c$ is a monomorphism. The injectivity of $I$ gives us a morphism $r$ with $r c=1_{l} . c$ is a reflection, so the equality $c r c=c$ implies $c r=1_{I}$. Therefore $c$ is an isomorphism and $I$ belongs to $\mathscr{X}$.

(ii) $\rightarrow$ (iii). Trivial.

(iii) $\rightarrow$ (i). Let $\mathscr{I}$ be the class of injective objects of $\mathscr{K}$ which belong to $\mathscr{X}$. Lemma 2 implies that $L \mathscr{I}$ is a reflective subcategory with a monofunctor as reflector. But $\mathrm{SL} \mathscr{G}=\mathrm{S} \mathscr{X}$, so the assertion follows from Theorem 1 . 
Property (ii) is hereditary with respect to intersections. Therefore we get the following theorem as an immediate consequence of the equivalence of (i) and (ii) in Theorem 3:

THEOREM 4. Let $\mathscr{K}$ be a well-powered and co-well-powered complete category with injective envelopes. If $\left\{\mathscr{X}_{i} ; i \in I\right\}$ is any class of elements of $\boldsymbol{M}$, and $\mathscr{X}$ is the (set-theoretical) intersection of the $\mathscr{X}_{i}$, then $\mathscr{X}$ is an element of $M$.

5. Locally minimal and locally maximal elements of $M$. Let $\mathscr{K}$ be a wellpowered and co-well-powered complete category with injective envelopes. If we look at all reflective subcategories $\mathscr{X}$ with fixed $\mathscr{L}=\mathrm{s} \mathscr{X}$, there is a biggest subcategory, namely $\mathscr{L}$, but there is also a smallest subcategory: the limit-closure of the injective objects of $\mathscr{K}$ which belong to $\mathscr{L}$. The following theorem characterizes these subcategories. We start with the category $\mathscr{L}$ and denote by $M_{0}$ the class of all reflective subcategories $\mathscr{X}$ of $\mathscr{L}$ with $\mathrm{s} \mathscr{X}=\mathscr{L}$.

THEOREM 5. Let $\mathscr{L}$ be a well-powered complete category with injectives. If $\mathscr{X}$ is a limit-closed subcategory, the following assertions are equivalent:

(i) $\mathscr{X}$ is the limit-closure of the injectives of $\mathscr{L}$,

(ii) $\mathscr{X}$ is the smallest element of $M_{0}$,

(iii) $\mathscr{X}$ belongs to $\boldsymbol{M}_{0}$ and has no proper bimorphisms.

Proof. (i) $\leftrightarrow$ (ii). Let $\mathscr{I}$ be the class of all injectives of $\mathscr{L}$. $\mathscr{L}$ has enough injectives, therefore we have $\operatorname{s} \mathscr{I}=\mathscr{L}$, but then a fortiori $\operatorname{sL}(\mathscr{I})=\mathscr{L}$. Lemma 1 implies that $\mathrm{L}(\mathscr{I})$ belongs to $\boldsymbol{M}_{0}$. If $\mathscr{X}$ is any element of $\boldsymbol{M}_{0}$, then $\mathscr{X}$ contains all injective objects of $\mathscr{L}$; given an injective object $I$, we look at the reflection $c: I \rightarrow I^{\prime}$ of $I$ into $\mathscr{X} . \mathscr{X}$ is monoreflective, therefore $c$ is a monomorphism and the injectivity of $I$ implies that $c$ is a coretraction. But monoreflective subcategories are epireflective, so $c$ is also an epimorphism and therefore an isomorphism. So we have shown that $\mathscr{I}$, and therefore $L \mathscr{I}$, is contained in $\mathscr{X}$.

(i) $\rightarrow$ (iii). We first observe that the limit-closure $\mathrm{L} \mathscr{F}$ of the injectives of $\mathscr{L}$ is just the full subcategory $\mathscr{X}$ of all objects $X$ for which there exists an equalizerdiagram

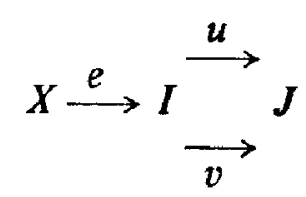

where $I$ and $J$ are injective. Clearly, $\mathscr{X}$ is contained in $L \mathscr{I}$. So we have to show that $\mathscr{X}$ is limit-closed. But $\mathscr{I}$ is product-closed, so it is easy to see that $\mathscr{X}$ is productclosed. It remains to verify that $\mathscr{X}$ is closed under the formation of equalizerdiagrams

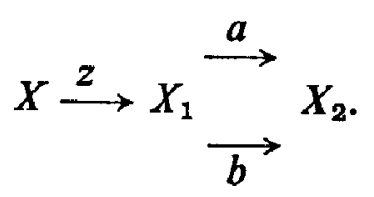


For every $X_{i}$ we have equalizer-diagrams of the form $\left(^{*}\right)$, say

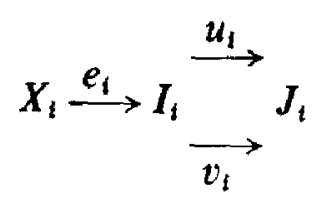

where $I_{i}$ and $J_{i}$ are injective. In particular, $I_{2}$ is injective and $e_{1}$ is a monomorphism, so we can find morphisms $a^{\prime}: I_{1} \rightarrow I_{2}$ and $b^{\prime}: I_{1} \rightarrow I_{2}$ with $a^{\prime} e_{1}=e_{2} a$ and $b^{\prime} e_{1}=e_{2} b$. One verifies that the morphism $e_{1} z$ is the limit of the following diagram

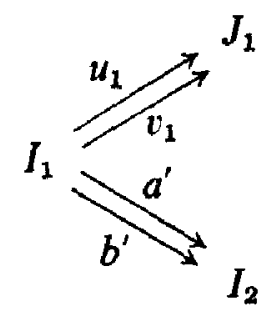

and this implies that there exists a diagram of the form $\left(^{*}\right)$ for $X$. (Look at the usual construction of limits by means of products and equalizers.)

Now given a bimorphism $b: X \rightarrow Y$ in $\mathscr{X}=\mathrm{L} \mathscr{\emptyset}$, we take an equalizer-diagram of the form $\left({ }^{*}\right)$, where $I$ and $J$ are injective. $b$ is a monomorphism, $I$ is injective, so there exists a morphism $d$ with $d b=e$. But equalizers are extreme monomorphisms, so the epimorphism $b$ must be an isomorphism. So all bimorphisms in $L \mathscr{I}$ are isomorphisms.

(iii) $\rightarrow$ (ii). We show that a subcategory $\mathscr{X}$ with the properties of (iii) is a minimal element of $\boldsymbol{M}_{0}$. Given an element $Y$ of $\boldsymbol{M}_{0}$ which is contained in $\mathscr{X}$. The equality $\mathbf{s} \mathscr{Y}=\mathscr{L}$ implies that $\mathscr{Y}$ is a monoreflective and therefore bireflective subcategory of $\mathscr{L}$. But then $\mathscr{Y}$ is also a bireflective subcategory of $\mathscr{X}$. All bimorphisms of $\mathscr{X}$ are isomorphisms, so the reflections of $\mathscr{X}$ into $\mathscr{Y}$ are in fact isomorphisms and $\mathscr{X}=\mathscr{Y}$.

We call a reflective subcategory $\mathscr{X}$ locally minimal (maximal) iff $\mathscr{X}$ belongs to $\boldsymbol{M}$ and $\mathscr{X}$ is minimal (maximal) in the class of reflective subcategories with fixed $s \mathscr{X}$. Thus the locally minimal subcategories of $\mathscr{K}$ are those of the form $L \mathscr{F}$, the locally maximal subcategories are those of the form SL $\mathscr{I}$, where $\mathscr{I}$ is a class of injective objects. In a category without proper bimorphisms, another characterization can be given using the following theorem:

THEOREM 6. Let $\mathscr{K}$ be a well-powered and co-well-powered complete category with injectives. A reflective subcategory is locally minimal if and only if the reflector maps monomorphisms onto regular monomorphisms $\left({ }^{5}\right)$.

Proof. We need the following result which is essentially due to Kelly [7]:

(5) A monomorphism $m$ is regular, iff $m$ is the equalizer of two morphisms. 
LEMMA 3. Let $\mathscr{L}$ be a well-powered complete category with amalgamations. Then every morphism $x$ may be factorized as $x=m e$ where $e$ is an epimorphism and $m$ is a regular monomorphism.

Proof of the lemma. (a) Every regular monomorphism is the equalizer of two monomorphisms. If $m$ is the equalizer of $a_{1}: X \rightarrow Y(i=1,2)$, we take the product $Z$ of $X$ and $Y$ with the projections $x: Z \rightarrow X$ and $y: Z \rightarrow Y$, and define $a_{i}^{\prime}$ by $x a_{i}^{\prime}=1_{x}$ and $y a_{i}^{\prime}=a_{i}$. The last equation shows that $a_{1}^{\prime}$ is a monomorphism, and it is easy to see that $m$ is the equalizer of $a_{1}^{\prime}$ and $a_{2}^{\prime}$.

(b) The composition of two regular monomorphisms is a regular monomorphism. Let $m: X \rightarrow Y$ be the equalizer of $a_{i}: Y \rightarrow A$, and $n: Y \rightarrow Z$ be the equalizer of $b_{i}: Z \rightarrow B(i=1,2)$. We can assume that the $a_{1}$ are monomorphisms. The existence of amalgamations gives us a commutative diagram, where all morphisms are monomorphisms:

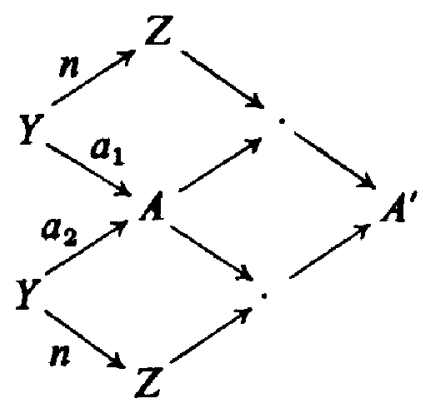

Therefore we have monomorphisms $n^{\prime}: A \rightarrow A^{\prime}$ and $a_{i}^{\prime}: Z \rightarrow A^{\prime}$ with $a_{i}^{\prime} n=n^{\prime} a_{i}$ $(i=1,2)$. We define $C$ as the product of $A^{\prime}$ and $B$ with the projections $p_{A^{\prime}}: C \rightarrow A^{\prime}$ and $p_{B}: C \rightarrow B$, and for $i=1,2$ we define $c_{i}: Z \rightarrow C$ by $p_{A^{\prime}} c_{i}=a_{i}^{\prime}$ and $p_{B} c_{i}=b_{i} . \mathrm{nm}$ is the equalizer of $c_{1}$ and $c_{2}$ : we have the equality $c_{1} n m=c_{2} n m$, and given $u$ with $c_{1} u=c_{2} u$, we have

$$
b_{1} u=p_{B} c_{1} u=p_{B} c_{2} u=b_{2} u,
$$

and therefore we can factorize $u$ as $u=n u^{\prime}$. Now the equality

$$
n^{\prime} a_{1} u^{\prime}=a_{1}^{\prime} n u^{\prime}=a_{1}^{\prime} u=p_{A^{\prime}} c_{1} u=p_{A^{\prime}} c_{2} u=a_{2}^{\prime} n u^{\prime}=n^{\prime} a_{2} u^{\prime}
$$

implies $a_{1} u^{\prime}=a_{2} u^{\prime}$, because $n^{\prime}$ is a monomorphism. Therefore we can factorize $u^{\prime}$ as $u^{\prime}=m u^{\prime \prime}$, and $u=(\mathrm{nm}) u^{\prime \prime}$ gives us the factorization we have looked for.

(c) Given an arbitrary morphism $x: A \rightarrow B$ in $\mathscr{L}$, take the smallest subobject $m$ of $B$ which is a regular monomorphism and through which $x$ factors, say $x=m e$. $m$ exists, because our assumptions imply that the regular monomorphisms are closed under intersections. From (b) it follows that $e$ is an epimorphism. This proves the lemma.

If $\mathscr{X}$ is locally minimal, then, according to Theorem $5, \mathscr{X}$ has no proper bimorphisms. $\mathscr{X}$ satisfies the conditions of Lemma 3 , so every monomorphism of $\mathscr{X}$ is a regular monomorphism, and the reflector $S: \mathscr{K} \rightarrow \mathscr{X}$ maps monomorphisms to 
regular monomorphisms. Conversely, if $\mathscr{X}$ is reflective and the reflector $S: \mathscr{X} \rightarrow \mathscr{X}$ maps monomorphisms onto regular monomorphisms, $S$ is, in particular, a monofunctor and so $\mathscr{X}$ belongs to $\boldsymbol{M}$. If $b$ is a bimorphism of $\mathscr{X}, b$ is a monomorphism of $\mathscr{X}$, so $b=S(b)$ is a regular monomorphism of $\mathscr{X}$. But a morphism which is an epimorphism and a regular monomorphism must be an isomorphism. So $\mathscr{X}$ has no proper bimorphisms and $\mathscr{X}$ is locally minimal.

COROLlary. Let $\mathscr{X}$ be a well-powered and co-well-powered complete category with injectives and without proper bimorphisms. Then

(a) $A$ reflective subcategory is locally minimal if and only if the reflector preserves regular monomorphisms.

(b) A reflective subcategory is locally maximal if and only if it is epireflective and the reflector preserves monomorphisms.

Proof. (a) Lemma 3 implies that every monomorphism of $\mathscr{X}$ is a regular monomorphism, so (a) is a consequence of Theorem 6. (b) In a well-powered and co-well-powered complete category without bimorphisms the epirefiective subcategories are just those limit-closed subcategories $\mathscr{X}$ which satisfy the equality $\mathscr{X}=\mathbf{s} \mathscr{X}[11]$.

RemarK. If $\mathscr{X}$ is a reflective subcategory of $\mathscr{X}$ and the reflector $S: \mathscr{K} \rightarrow \mathscr{X}$ preserves regular monomorphisms, then $S$ preserves equalizers of cokernel pairs. For given a cokernel pair $q_{1}, q_{2}$ (of a morphism $x$ ), and the equalizer $m$ of $q_{1}$ and $q_{2}$, then $q_{1}, q_{2}$ is also the cokernel pair of $m$, that is

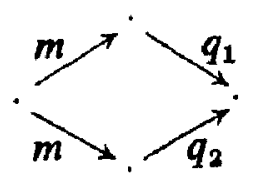

is a pushout diagram. But as a left adjoint, $S$ preserves pushouts, so

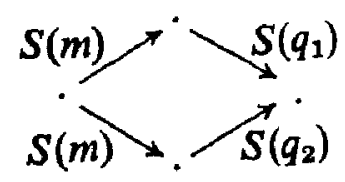

is a pushout diagram in $X$, and $S\left(q_{1}\right), S\left(q_{2}\right)$ is the cokernel pair of $S(m)$. But $S(m)$ is a regular monomorphism, so $S(m)$ is the equalizer of this cokernel pair: $S(m)$ is the equalizer of $S\left(q_{1}\right)$ and $S\left(q_{2}\right)$. If $\mathscr{K}$ has cokernel pairs, $S$ preserves regular monomorphisms if and only if $S$ preserves equalizers of kernel pairs.

6. Applications. (a) Module categories. Let $R$ be an associative ring with 1 and $\mathfrak{M}_{R}$ be the category of all $R$-modules. In studying the localization of rings the concept of a (hereditary) torsion-theory $(\mathscr{P}, 2)[2]$, [9] has been useful. As Gabriel [4] has shown, every torsion-theory $(\mathscr{P}, \mathscr{Q})$ determines a reflective subcategory $\mathscr{E}$ with $\mathscr{Q}=\mathbf{S} \mathscr{E}$ and with an exact functor as reflector. Both $\mathscr{Q}$ and $\mathscr{E}$ are reflective 
subcategories with a monofunctor as reflector and the following proposition shows that the elements of $\boldsymbol{M}$ are just those limit-closed subcategories $\mathscr{X}$ with $\mathscr{E} \subseteq \mathscr{X} \subseteq \mathscr{Z}$ for a suitable torsion-theory (see also [12]).

Proposition 1. For a limit-closed subcategory $\mathscr{X}$ of $\mathfrak{M}_{R}$ the following properties are equivalent:

(i) $\mathscr{X}$ is reflective and has a monofunctor as reflector,

(ii) there is a torsion-theory $(\mathscr{P}, \mathscr{2})$ with $\mathrm{s} X=\mathscr{Q}$,

(iii) there is a reflective subcategory $\mathscr{E}$ with an exact reflector and $\mathscr{E} \subseteq \mathscr{X} \subseteq \mathrm{S} \mathscr{E}$.

Proof. (i) $\rightarrow$ (ii). According to Theorem 3, $X$ is closed under essential extensions, therefore $\mathbf{s} \mathscr{X}$ is closed under products, subobjects and essential extensions, and it is well known (see for example [13]) that these properties characterize the class 2 of a torsion-theory $(\mathscr{P}, \mathscr{Q})$.

(ii) $\rightarrow$ (iii). Take for $\mathscr{E}$ the reflective subcategory $\mathscr{E}$ with $\mathscr{Q}=\mathbf{S} \mathscr{E}$ and with an exact reflector constructed by Gabriel. Then $\mathscr{X} \subseteq \mathrm{S} \mathscr{E}$ and we have to show that $\mathscr{E}$ is contained in $\mathscr{X}$. But $\mathscr{E}$ is an abelian category and contains therefore no proper bimorphism. Applying Theorem 5 for $\mathscr{L}=2$ shows that $\mathscr{E}$ is contained in every reflective subcategory $\mathscr{Y}$ with $\mathrm{s} \mathscr{Y}=\mathscr{2}$. But $\mathscr{Q}$ has a monofunctor as reflector, so $\mathscr{X}$ is in fact reflective (Theorem 1), and $\mathscr{E}$ is contained in $\mathscr{X}$.

(iii) $\rightarrow$ (i). We have the equality $\mathrm{s} \mathscr{E}=\mathrm{s} \mathscr{T}$ and $\mathscr{E}$ has a monofunctor as reflector, so the assertion is a consequence of Theorem 1 . The locally maximal elements of $\boldsymbol{M}$ are just the subcategories $\mathscr{Q}$, where $(\mathscr{P}, \mathscr{Q})$ is a torsion-theory, the locally minimal elements are the reflective subcategories with an exact reflector $(=$ the localizations of Gabriel). It is easy to see that in general these are not the only elements of $\boldsymbol{M}$ for example in the category $\mathfrak{M}_{z}$ of all abelian groups the full subcategory of the $p$-divisible torsionfree groups (where $p$ is a prime) is a reflective subcategory with a monofunctor as reflector, but is neither locally minimal nor locally maximal. This is a special case of the following construction:

Proposition 2. Let $(\mathscr{P}, \mathscr{2})$ be a torsion-theory in $\mathfrak{M}_{R}$ and $\mathscr{P}^{\prime}$ be a subcategory of $\mathscr{P}$. Then the full subcategory $\mathscr{X}$ of all objects $X$ of $Q$ with $\operatorname{Ext}(P, X)=0$ for all $P \in \mathscr{P}^{\prime}$ is a reflective subcategory with a monofunctor as reflector.

Proof. We only have to show that $\mathscr{X}$ is limit-closed. $\mathscr{X}$ is product-closed, so we consider the case where $k: K \rightarrow X_{1}$ is the kernel of $x: X_{1} \rightarrow X_{2}$, where $x$ is a morphism in $\mathscr{X}$. If $x=m e$ is the factorization of $x$ in an epimorphism $e$ and a monomorphism $m$, the exact sequence

$$
0 \longrightarrow K \stackrel{k}{\longrightarrow} X_{1} \stackrel{e}{\longrightarrow} Q \longrightarrow 0
$$

determines an exact sequence

$$
0 \rightarrow \operatorname{Hom}(P, K) \rightarrow \operatorname{Hom}\left(P, X_{1}\right) \rightarrow \operatorname{Hom}(P, Q) \rightarrow \operatorname{Ext}(P, K) \rightarrow \operatorname{Ext}\left(P, X_{1}\right) .
$$


$Q$ is a subobject of $X_{2}$ in $\mathscr{X}$, so belongs to $\mathscr{Q}, P$ is an object of $\mathscr{P}$, therefore we have $\operatorname{Hom}(P, Q)=0$. Ext $\left(P, X_{1}\right)=0$ now implies $\operatorname{Ext}(P, K)=0$.

(b) Functor-categories. Let $\mathscr{G}$ be a small category and $[\mathscr{C}, \Im]$ the category of all functors from $\mathscr{C}$ into the category $\Xi$ of sets. If $\mathrm{t}$ is a Grothendieck-topology on $\mathscr{C}$, we denote by $\mathscr{Q}_{t}$ the full subcategory of the separated presheaves, by $\mathscr{E}_{t}$ the full subcategories of all sheaves with respect to $t$ [14]. Both $\mathscr{Q}_{\mathfrak{t}}$ and $\mathscr{E}_{t}$ are reflective subcategories with monofunctors as reflectors and the elements of $\boldsymbol{M}$ are just those limit-closed subcategories $\mathbb{X}$ with $\mathscr{E}_{1} \subseteq \mathscr{X} \subseteq \mathscr{Q}_{1}$ for a suitable topology:

Proposition 3. For a limit-closed subcategory $\mathscr{X}$ of $[\mathscr{C}, \mathbb{S}]$ the following properties are equitalent:

(i) $\mathscr{X}$ is reflective and has a monofunctor as reflector,

(ii) $\mathrm{s} X=\mathcal{2}_{\mathrm{t}}$ for a Grothendieck-topology $\mathrm{t}$,

(iii) there is a reflective subcategory $\mathscr{E}$ with a reflector respecting finite limits and $\mathscr{E} \subseteq \mathbb{X} \subseteq \mathbf{S}$.

The proof is quite similar to (a) and will be omitted.

The locally maximal elements of $\boldsymbol{M}$ are just the categories of the separated presheaves, the locally minimal elements are the categories of all sheaves with respect to a topology.

(c) Categories of topological spaces. We call a full subcategory of the category $\mathfrak{I}$ of all topological spaces a category of topological spaces. There are some categories $\mathscr{K}$ of topological spaces which satisfy the assumptions of Theorem 1: The category of all $T_{1}$-spaces and the category $\mathfrak{T}$ itself both have amalgamations. However there are only trivial subcategories of the form $\mathrm{s} X$ which have a monofunctor as reflector:

Proposition 4. Let $\mathscr{X}$ be a category of topological spaces which contains all discrete spaces. A reflective subcategory with a monofunctor as reflector is either monoreflective or contains only spaces with at most one point.

Proof. We may assume that the subcategory $\mathscr{X}$ has a monofunctor as reflector and contains a space $X$ with more than one point. First we notice that for a discrete space $D$ the reflection morphism $r_{D}: D \rightarrow D^{\prime}$ into $\mathscr{X}$ is a monomorphism: for given two distinct points $d_{1}$ and $d_{2}$ in $D$ we find a (set-theoretical) map $D \stackrel{x}{\rightarrow} X$ which does not identify $d_{1}$ and $d_{2}$. But $D$ is discrete, so $x$ is continuous. Therefore we can factorize $x$ through $r_{D}$, and $r_{D}$ does not identify $d_{1}$ and $d_{2}$. Now for an arbitrary space $K$ in $\mathscr{H}$ we find a bijective map $b: D \rightarrow K$ where $D$ is discrete. We have the equality $r_{K} b=R(b) r_{D}$ where $r_{K}$ and $r_{D}$ are the refiection morphisms and $R: \mathscr{H} \rightarrow \mathscr{X}$ is the reflector. $R$ is a monofunctor, so $R(b)$ is a monomorphism. This implies that $r_{K} b$ is a monomorphism. $b$ is bijective, so $r_{K}$ is a monomorphism.

(d) Categories with only coretractions as monomorphisms. If every monomorphism of $\mathscr{K}$ is a coretraction, then every functor $\mathscr{K} \rightarrow \mathscr{L}$ (where $\mathscr{L}$ is an arbitrary category) is a monofunctor. In this case $\boldsymbol{M}$ is the class of all reflective subcategories. 
The assumption is verified in every abelian category of global dimension 0 , for example in the module-categories $\mathfrak{R}_{R}$ where $R$ is (artinian) semisimple. However, if in $\mathscr{X}$ every monomorphism is a coretraction, the same assertion is true in every full subcategory, and so every reflective subcategory is even epireflective.

\title{
REFERENCES
}

1. S. Baron, Reflectors as compositions of epi-reflectors, Trans. Amer. Math. Soc. 136 (1969), 499-508. MR 38 \#4535.

2. S. E. Dickson, A torsion theory for Abelian categories, Trans. Amer. Math. Soc. 121 (1966), 223-235. MR 33 \#162.

3. Ph. Dwinger, The amalgamarion problem from a categarical point of view, Proc. Conference on Universal Algebra, Queen's Paper 25, Kingston, Ont., 1970.

4. P. Gabriel, Des catégories abéliennes, Bull. Soc. Math. France 90 (1962), 323-448. MR 38 \#1144.

5. H. Herrlich, Topologische Reflexionen und Corefiexionen, Lecture Notes in Math., no. 78 , Springer-Verlag, Berlin and New York, 1968. MR 41 \#988.

6. - An example in category theory, Math. Z. 113 (1970), 309-312.

7. G. M. Kelly, Monomorphisms, epimorphisms, and pull-backs, J. Austral. Math. Soc. 9 (1969), 124-142. MR 39 \#1515.

8. J. F. Kennison, Full reflective subcategories and generalized covering spaces, Illinois J. Math. 12 (1968), 353-365. MR 37 \#2832.

9. J. Lambek, Torsion theories, additive semantics, and rings of quotients, McGill University, Montreal, 1970.

10. B. Mitchell, Theory of categories, Pure and Appl. Math., vol. 17, Academic Press, New York, 1965. MR 34 \#2647.

11. G. Preuss, Eine Charakterisierung epireflektiver Unterkategorien einer Kategorie mit Inversionseigenshaft, Manuscripta Math. 1 (1969), 307-316. MR 41 \#1831.

12. C. M. Ringel, Diagonalisierungspaare. II, Math. Z. 122 (1971), 10-32.

13. R. G. Swan, Algebraic K-theory, Lecture Notes in Math., no. 76, Springer-Verlag, Berlin and New York, 1968. MR 39 \#6940.

14. J. L. Verdier, Séminaire géométrie algébrique 1963/64, Inst. Hautes Études Sci., Paris, 1964. MR 34 \#7520.

\author{
Universität Tübingen, Federal Republic of Germany \\ Carleton University, Ottawa, Canada
}

